\title{
Preferência podal em lactentes com síndrome de Down: fatores extrínsecos e intrínsecos
}

\author{
Foot preference in infants with Down syndrome: extrinsic and intrinsic factors
}

Gabriela Lopes dos Santos¹, Thaís Bortolini Bueno², Eloisa Tudella³, Jadiane Dionísio 4

RESUMO I Durante o processo de aquisição do chute em lactentes, a preferência podal pode ser influenciada de forma diferente pelos fatores extrínsecos e intrínsecos. O objetivo foi comparar a preferência podal e verificar a influência do peso adicional e do Reflexo Tônico Cervical Assimétrico (RTCA) na preferência podal entre lactentes com síndrome de Down e típicos. Participaram do estudo cinco lactentes com síndrome de Down e cinco típicos aos três e quatro meses de idade. O experimento foi subdividido em quatro condições experimentais: Treinamento, Linha de Base, Peso e Pós-Peso. Os lactentes com síndrome de Down apresentaram preferência à esquerda ou indefinida e os típicos, preferência à direita. $O$ RTCA influenciou na preferência podal em ambos os grupos, indicando que o lado de rotação da cabeça orientou o chute do mesmo lado. Nos lactentes com síndrome de Down a correlação entre RTCA e chutes foi positiva, sendo forte aos três meses e moderada aos quatro meses. Para os lactentes típicos foi observada correlação positiva fraca aos três meses, e aos quatro meses a correlação não foi estatisticamente significativa. Lactentes com síndrome de Down apresentam preferência podal contrária aos lactentes típicos. O RTCA exerce influência na preferência podal por um período mais longo em lactentes com síndrome de Down em comparação aos lactentes típicos.

Descritores I desenvolvimento infantil; reflexo; lateralidade funcional.
ABSTRACT I During the acquisition process of the kick in infants, foot preference may be influenced differently by extrinsic and intrinsic factors. The aim was to compare the foot preference and find the influence of additional weight and Asymmetrical Neck Tonic Reflex (ANTR) on the foot preference of infants with Down syndrome and typical ones. Five infants with Down syndrome and five typical infants at three and four months of age participated of the sudy. The experiment was divided into four experimental conditions: Training, Baseline, Weight and After Weight. Infants with Down syndrome showed preference to the left or indefinite, the typical infants showed preference to the right. ANTR influenced the foot preference in both groups, indicanting that the rotation of head side oriented the kicking for the same side. For infants with Down syndrome the correlation between ANTR and kicking was positive, with a strong positive correlation at three months of age and a moderate positive correlation at four months of age observed. For typical infants it was observed weak positive correlation at three months of age and at four months of age, the correlation was not significant. Infants with Down syndrome have foot preference contrary to typical infants. ATNR influences the foot preference for a longer period in infants with Down syndrome compared to typical infants.

Keywords I child development; reflex; functional laterality.

Estudo desenvolvido no Laboratório de Pesquisa e Análise de Movimento (LaPAM), Departamento de Fisioterapia, Universidade Federal de São Carlos (UFScar) - São Carlos, SP, Brasil.

'Mestranda pelo Departamento de Fisioterapia da Universidade Federal de São Carlos (UFSCar) - São Carlos(SP), Brasil. 2Fisioterapeuta da Clínica Fit e Fisioterapia - São Paulo (SP), Brasil.

${ }^{3}$ Doutora em Psicologia (Psicologia Experimental); Professora do curso de Fisioterapia da UFSCar - São Carlos (SP), Brasil. ${ }^{4}$ Mestre em Fisioterapia; Professora da Faculdade de Educação Física e Fisioterapia na Universidade Federal de Uberlândia (UFU) Uberlândia (MG), Brasil.

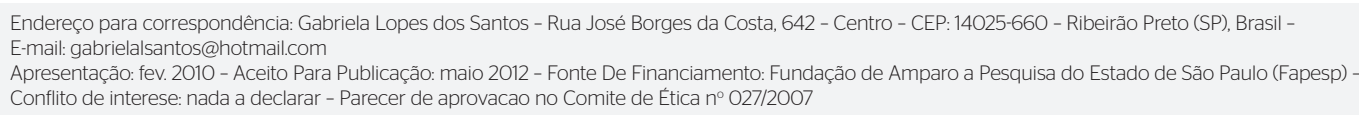




\section{INTRODUÇÃO}

Preferência lateral ou lateralidade é caracterizada pelo uso preferencial de um membro em uma ação voluntária ${ }^{1}$, sendo a preferência manual a expressão mais observada desse processo de lateralização, seguida da preferência podal ${ }^{2}$. Esta preferência podal pode ser observada nas primeiras semanas de vida (4 a 24 semanas) nos movimentos de chutes de lactentes típicos, sendo essa preferência, em sua maioria, à direita ${ }^{3}$. Além disso, outros estudos observaram que essa preferência podal à direita se manteve ao longo dos anos ${ }^{2,4,5}$.

Os movimentos de chutes são considerados um dos comportamentos motores mais precoces em lactentes, podendo ser observado desde a vida intrauterina. Em recém-nascidos, esses movimentos são caracterizados por flexão e extensão total das articulações de quadril, joelho e tornozelo, denominados chutes em-fase (em bloco), sendo considerados como padrão imaturo de movimento ${ }^{6}$. Ao longo do desenvolvimento, o chute passa a ser realizado combinando flexão de uma articulação com extensão da outra, tornando-se um movimento mais maturo, denominado fora-de-fase. Com esse padrão mais desenvolvido de chute, o lactente, futuramente, será capaz de realizar tarefas mais complexas como engatinhar, escalar e andar ${ }^{3,7,8}$. No entanto, em lactentes com síndrome de Down há um atraso no processo de desenvolvimento do chute e, consequentemente, um atraso na aquisição de marcos motores importantes, tais como o engatinhar e a marcha ${ }^{9}$, devido às alterações neuromotoras, como hipotonia muscular, hiperextensibilidade articular, distúrbio no mecanismo de controle postural e equilíbrio ${ }^{10,11}$. Além das alterações neuromotoras, as crianças com Síndrome de Down também apresentam alterações na organização cerebral $^{12}$, o que pode levar ao desenvolvimento de uma lateralidade à esquerda, inconsistente e/ou cruzada ${ }^{13,14}$.

Contudo, tanto em lactentes típicos quanto com síndrome de Down, todas essas mudanças que ocorrem no padrão de movimento do chute e no desenvolvimento da preferência podal sofrem influência de elementos do organismo e da especificidade da tarefa ${ }^{15,8}$, em virtude da interação de fatores intrínsecos (restrições orgânicas) e extrínsecos (restrições ambientais) ${ }^{15,16}$.

Utilizando o peso adicional em um dos membros inferiores de lactentes a termo, com seis semanas de idade, Thelen, Skala e Kelso (1987) observaram que a amplitude e velocidade do movimento foram mantidas na perna com peso, porém, houve um aumento na perna sem peso. Esse achado demonstra que os lactentes perceberam o peso adicional e se ajustaram, causando, assim, um aumento no nível da ativação neural. Ulrich, Ulrich, Ângulo-Kinzler e Chapman ${ }^{17}$, com o objetivo de observar a influência do peso adicional nos membros inferiores de lactentes a termo, concluíram que lactentes que apresentaram melhor performance com o peso adicional, apresentaram melhor desempenho nas funções motoras de engatinhar e caminhar, além de serem adquiridas de forma mais rápida.

Assim como o peso adicional, fatores intrínsecos como, por exemplo, o reflexo tônico cervical assimétrico (RTCA) podem influenciar na preferência podal de lactentes ${ }^{3,18}$. O RTCA é observado quando se roda a cabeça do lactente para um dos lados e como resposta obtém-se a abdução e flexão dos membros superior e inferior do lado occipital e abdução e extensão dos membros superior e inferior do lado face. No entanto, essas respostas ocorrem com menor intensidade nos membros inferiores. Em lactentes a termo esse reflexo é claramente evidenciado aos dois meses, enquanto aos quatro meses de idade é raramente observado ${ }^{19}$. Há evidências de que, quando o lactente encontra-se em RTCA, mais movimentos são produzidos pelo membro inferior do lado facial. Além disso, em lactentes com desenvolvimento típico com 4 a 24 semanas de idade são observados com maior frequência RTCA para o lado direito, aumentando a frequência de chutes com o membro inferior direito ${ }^{3,18}$.

Diante do exposto, verifica-se que fatores intrínsecos e extrínsecos podem influenciar os movimentos de chutes dos lactentes, incluindo a preferência podal. No entanto, a influência dessas variáveis no comportamento do chute é pouco observada na literatura atual, especialmente em lactentes com síndrome de Down. Com isso, ressalta-se a necessidade de um estudo que compare a influência dos fatores extrínsecos e intrínsecos na preferência podal desses lactentes.

Portanto, o presente estudo tem como objetivo comparar a preferência podal e verificar a influência dos fatores extrínseco (peso adicional de $1 / 3$ da massa do membro inferior) e intrínseco (RTCA) na preferência podal de lactentes com síndrome de Down e típicos aos três e quatro meses de idade.

Para tanto, foram testadas as seguintes hipóteses: 1) Lactentes com síndrome de Down apresentam preferência podal à esquerda e lactentes típicos à direita aos três e quatro meses de idade; 2) $\mathrm{O}$ peso adicional influencia na preferência podal de lactentes com síndrome de Down e típicos aos três e quatro meses de idade; 
e 3) O RTCA influencia na preferência podal dos lactentes com Síndrome de Down aos três e quatros meses de idade, e dos lactentes típicos somente aos três meses.

\section{METODOLOGIA}

Este estudo de caráter longitudinal foi aprovado pelo Comitê de Ética e Pesquisas em Seres Humanos da Universidade Federal de São Carlos (UFSCar), sob o Parecer no 027/2007.

\section{Amostra}

Participaram do estudo 10 lactentes saudáveis, de ambos os sexos, com três e quatro meses de idade, subdivididos em dois grupos: semiexperimental, composto por 5 lactentes com síndrome de Down com idade gestacional média de 36,20 semanas $( \pm 0,45)$, peso ao nascimento médio de 2,56 quilogramas $( \pm 0,23)$, comprimento ao nascimento médio de 46,1 centímetros $( \pm 2,84)$ e índice de Apgar médio 8,52 $( \pm 0,45)$ no primeiro minuto e 9,00 $( \pm 0,00)$ no quinto minuto; e Controle: 5 lactentes típicos com idade gestacional média de 37,60 semanas $( \pm 0,55)$, peso ao nascimento médio 3,75 de quilogramas $( \pm 0,24)$, e comprimento ao nascimento médio de 48,6 centímetros $( \pm 0,55)$ e Apgar de médio de $9,00( \pm 0,00)$ e $9,8( \pm 0,45)$ no primeiro e quinto minuto, respectivamente.

Foram excluídos do estudo os lactentes com alterações ortopédicas, deficiências auditivas e sensoriais que pudessem prejudicar a realização da tarefa. Os desligamentos dos lactentes do estudo ocorreram quando faltaram em qualquer uma das duas avaliações, ou quando os responsáveis desistiram de participar do estudo e àqueles os quais apresentaram intercorrências que poderiam prejudicar os resultados.

\section{Instrumentos}

Foi utilizada uma ficha de avaliação constando de dados de identificação, dados do nascimento, resumo da história pré, peri e pós-natal e informações relacionadas às condições clínicas, registro do estado comportamental e de saúde dos lactentes, bem como para suas medidas antropométricas.

O peso do lactente foi verificado por meio de uma balança pediátrica digital e sua altura por meio de uma régua antropométrica. As medidas de comprimento dos membros inferiores e dos seus segmentos foram efetuadas utilizando-se fita métrica.

Foram utilizadas tornozeleiras de forma a permitir a colocação ou retirada de bolinhas de chumbo, de acordo com a massa do membro inferior do lactente $(1 / 3$ da massa $)^{20}$, e marcadores retroreflexivos de $0,5 \mathrm{~cm}$ de diâmetro, e a fixação desses marcadores no lactente foi realizada com micropore ${ }^{21}$.

Os lactentes foram posicionados em uma mesa específica desenvolvida para o estudo, na extremidade inferior da mesa havia um painel que, ao ser elevado pelos pés do lactente, acionava um móbile fixado a uma altura de aproximadamente 30 centímetros acima do rosto, agindo como estímulo visual e auditivo para a realização dos chutes ${ }^{20}$. A posição do painel foi definida pela seguinte fórmula: [(distância do comprimento da perna) $x$ sen $\left.\left(30^{\circ}\right)\right]^{22}$.

Para registro das avaliações foram utilizadas duas câmeras digitais JVC (modelo GY DV-300) acopladas a tripés posicionadas anterolateralmente à cadeira, uma em cada lado, e dois iluminadores posicionados ao lado das câmeras e direcionados para a parede para não prejudicar a visualização da imagem capturada. A imagem capturada foi analisada utilizando o Sistema Dvideow 6.320.

\section{Procedimentos}

A coleta de dados foi realizada no laboratório, na data de aniversário, considerando um intervalo de aproximadamente cinco dias antes ou após essa data. No dia da avaliação todo procedimento foi explicado para os responsáveis e estes assinaram o Termo de Consentimento Livre e Esclarecido, autorizando a participação dos lactentes no estudo.

No laboratório, os lactentes foram despidos pela mãe e o examinador coletou as medidas antropométricas (peso e comprimento total do corpo, comprimento e circunferência das coxas e pernas e comprimento e largura dos pés) com a finalidade de estimar a massa de cada membro inferior de acordo com o método de Schneider e Zernicke ${ }^{23}$.

Após esses procedimentos, os lactentes foram posicionados em supino em uma mesa específica desenvolvida para o estudo ${ }^{20}$ e estabilizados pelos ombros por um auxiliar de pesquisa.

O experimento teve duração total de aproximadamente 5 minutos, subdivididos em quatro condições: 1) Treinamento $(T)$ : os pés dos lactentes foram colocados pelo examinador no painel para ativar o móbile, sendo realizado três vezes com cada membro separadamente e 
três vezes com os dois membros juntos, por três segundos cada; 2) Linha de Base (LB): os lactentes ficavam livres para realizar os chutes, podendo elevar o painel com os pés e acionar o móbile; 3) Peso (P): idêntica à condição anterior, entretanto, colocava-se nos tonozelos do lactente uma tornozeleira confeccionada de tecido pesando 1/3 da massa do membro inferior; 4) Pós-peso (PP): idêntica à LB. Em cada condição foi admitido o tempo de 1 minuto para a realização dos chutes e entre as condições 2, 3 e 4 havia um intervalo de 30 segundos para a colocação ou retirada das tornozeleiras. Em todo o procedimento, os lactentes permaneceram em estado de alerta inativo ou ativo ${ }^{24}$.

Após a captura das imagens, estas foram analisadas para que fosse realizada a definição das variáveis analisadas. Desta forma, chute foi definido como movimento de um ou ambos os membros inferiores dos lactentes, partindo da flexão total de quadril, joelho e tornozelo para extensão e retornando para a posição inicial ${ }^{8,20}$.

\section{Descrição das Variáveis}

A preferência podal foi determinada em função da frequência de chutes com cada membro inferior, que consistiu no número de vezes que o lactente realizou o chute com os membros inferiores direito e esquerdo. A preferência podal foi estimada por meio do índice de preferência, dado pela seguinte equação: $\mathrm{IP}=(\mathrm{D}-\mathrm{E}) /(\mathrm{D}+\mathrm{E})$, na qual $\mathrm{D}$ e $\mathrm{E}$ referem-se à frequência absoluta de chutes realizados com os membros inferiores direito e esquerdo, respectivamente. Para chutes unipodais foi atribuído um ponto para membro inferior que realizou o movimento.
Nos casos de chutes bipodais foi atribuído um ponto para cada membro inferior. Valores entre 0,21 e 1,0 representam preferência podal à direita; valores entre 0,20 e -0,20 representam preferência indefinida; e valores entre $-0,21$ e $-1,0$ representam preferência podal à esquerda.

O RTCA foi definido quando a face estava voltada para o mesmo lado do membro que realizou o chute ${ }^{3,18}$.

\section{Análise Estatística}

Para verificar possíveis diferenças na preferência podal em relação aos grupos, condições experimentais e idade, foi realizado o ANOVA Three-Way com medidas repetidas, utilizando o programa SPSS. Aplicou-se a correlação de Pearson para verificar se há relação entre as variáveis preferência podal e RTCA aos três e quatro meses de idade para cada grupo. Foi considerado um nível de significância de 5\%.

\section{RESULTADOS}

Em relação à variável preferência podal foi observada diferença significativa entre os grupos $(F(1)=0,58$; $\mathrm{p}<0,01$ ), sendo que lactentes com síndrome de Down apresentaram preferência à esquerda ou indefinida quando comparado aos lactentes típicos, os quais mostraram uma preferência à direita. Em contrapartida, não foi constatado diferença significativa entre as idades $(F(1)=0,02 ; p=0,879)$, e condições de testes $(F(2)=0,30$; $\mathrm{p}=0,740)$ em ambos os grupos (Figura 1 ).

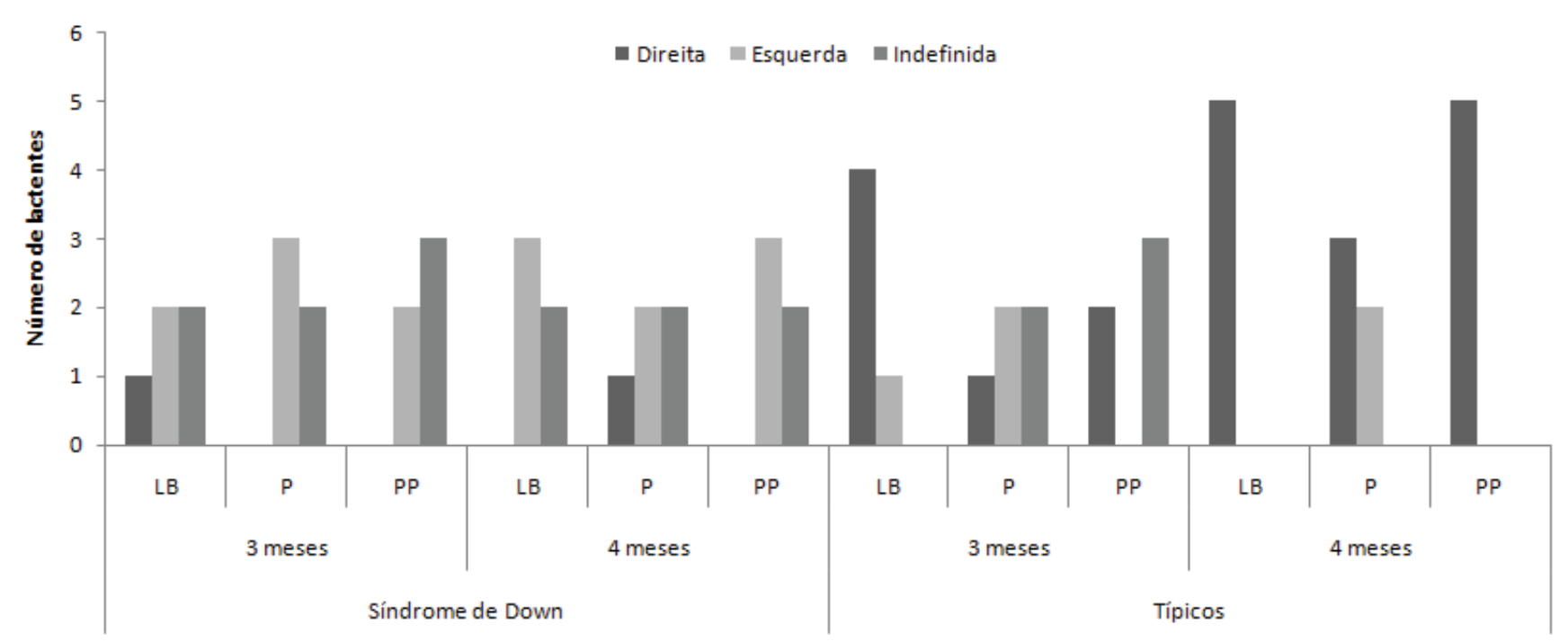

Figura 1. Preferência podal de lactentes com síndrome de Down e típicos nas condições experimentais Linha de Base (LB), Peso (P) e Pós-peso (PP) aos três e quatro meses de idade 
Tabela 1. Relação entre a preferência podal e o reflexo tônico cervical assimétrico em ambos os grupos aos 3 e 4 meses de idade

\begin{tabular}{|c|c|c|c|}
\hline & & $\begin{array}{l}\text { Coeficiente de } \\
\text { correlação (r) }\end{array}$ & $\begin{array}{l}\text { Significância } \\
\text { estatística (p) }\end{array}$ \\
\hline \multirow{2}{*}{ Síndrome de Down } & 3 meses & 0,893 & $<0,01$ \\
\hline & 4 meses & 0,524 & $<0,01$ \\
\hline \multirow{2}{*}{ Típicos } & 3 meses & 0,365 & $<0,01$ \\
\hline & 4 meses & 0,12 & 0,25 \\
\hline
\end{tabular}

Na relação preferência podal e RTCA foi observada forte correlação positiva aos três meses $(r=0,893$; $p<0,01)$ e aos quatro meses uma correlação positiva moderada $(\mathrm{r}=0,524 ; \mathrm{p}<0,01)$ para os lactentes com síndrome de Down (Tabela 1). Confirma-se este resultado através da análise qualitativa; $71,43 \%$ dos chutes realizados com o membro inferior esquerdo ocorreram quando o RTCA estava presente para o mesmo lado aos três meses de idade, e 57,83\% aos quatro meses de idade.

Para o grupo de lactentes típicos observou-se uma fraca correlação positiva aos três meses $(r=0,365$; $\mathrm{p}<0,01)$; contudo, aos quatro meses a correlação não foi significante $(r=0,117 ; p=0,252)$. Tal resultado é confirmado pela análise qualitativa, $45,92 \%$ dos chutes realizados com o membro inferior direito ocorreram quando o RTCA estava presente para o mesmo lado aos três meses de idade.

\section{DISCUSSÃO}

O presente estudo comparou a preferência podal de lactentes com síndrome de Down e típicos, observando diferença entre os grupos aos três e quatro meses de idade. Os lactentes com síndrome de Down apresentaram uma preferência podal à esquerda ou indefinida e os lactentes típicos demonstraram uma preferência à direita. Essa preferência à esquerda ou indefinida para o grupo com síndrome de Down pode ser justificada pelas alterações na organização cerebral ${ }^{12} \mathrm{e}$ em estruturas que desempenham papel importante no controle motor, tais como menor volume cerebral associado a uma redução na substância branca e cinzenta, hipoplasia do lobo frontal, temporal, cerebelo, tronco cerebral, além de anomalias na geometria do corpo caloso ${ }^{14,25}$.

No grupo com desenvolvimento típico a preferência podal à direita pode ser justificada pela perspectiva genética, a qual defende a ideia de que o hemisfério cerebral esquerdo é especializado em funções de linguagem, pensamento analítico, processamento seriado e controle sequenciado de atos motores ${ }^{26}$. Dessa forma, a preferência predominante pelo lado direito para o controle de ações motoras seria devido à maior aptidão do hemisfério cerebral esquerdo para controlar movimentos do lado contrário do corpo, em comparação com a capacidade de controle do hemisfério cerebral direito sobre o sistema muscular do lado esquerdo ${ }^{27}$.

Essa direção da preferência podal para ambos os grupos manteve-se ao longo do período analisado, não sendo observada diferença entre as idades, evidenciando que aos três e quatro meses de idade, lactentes já apresentam preferência por um dos lados. Assim, de acordo com os resultados, confirma-se a primeira hipótese do estudo, a qual afirma que lactentes com síndrome de Down apresentam uma preferência à esquerda e lactentes típicos, à direita, aos três e quatro meses de idade.

A preferência podal não foi influenciada pelo peso adicional para ambos os grupos aos três e quatro meses de idade. Para os lactentes com síndrome de Down, o peso adicional pode não ter influenciado devido à dificuldade de percepção do peso adicional pelos receptores presentes nos fusos musculares e tendões ${ }^{10}$. Esses receptores são denominados proprioceptores e apresentam como uma das funções a percepção do corpo no espaço que, por sua vez, associado ao tátil sinestésico e ao vestibular, influencia na formação do esquema corporal e na lateralidade ${ }^{28,29}$. Sendo assim, o peso, indiretamente, influenciaria na escolha de um dos membros; no entanto, devido a essa alteração apresentada pelos lactentes com síndrome de Down, estes não sofreram a influência do peso na preferência podal. Contudo, para o grupo de lactentes típicos, o peso pode ter sido insuficiente para estimular esses proprioceptores de maneira significativa. Dessa forma, de acordo com este resultado, refuta-se a segunda hipótese do estudo.

Em relação à influência do RTCA na preferência podal, para o grupo de lactentes típicos 45,92\% dos chutes realizados com o membro inferior direito ocorreram quando o RTCA estava presente para o mesmo lado somente aos três meses de idade e aos quatro meses de idade não foi constatada a influência do RTCA na preferência podal. Devido a isso, constata-se que o RTCA é mais evidente no segundo mês de idade e aos três meses torna-se menos intenso, levando a uma simetria pelo aumento do tônus da musculatura antigravitária, e aos quatro meses esse reflexo está ausente ${ }^{18}$. No entanto, para o grupo com Síndrome de Down foi observado que o RTCA influencia na preferência podal aos três $(71,43 \%)$ e aos quatro $(57,83 \%)$ meses de idade, evidenciando que as mudanças esperadas no comportamento do RTCA não foram observadas neste período para este 
grupo. Essa permanência do RTCA aos quatro meses de idade no grupo com síndrome de Down pode ser explicado pelo atraso no desenvolvimento que esses lactentes apresentam. Além disso, os lactentes desse estudos tinham idade gestacional média de 36,29 semanas, o que os classifica como pré-termo e também justifica o atraso no desenvolvimento e a permanência do RTCA aos quatro meses de idade. Deste modo, ratifica-se a primeira hipótese proposta, a de que o RTCA influencia a preferência podal dos lactentes com síndrome de Down aos três e quatro meses de idade e dos lactentes típicos somente aos três meses de idade.

\section{LIMITAÇÕES DO ESTUDO}

As limitações do estudo incluem o tamanho pequeno da amostra de dez lactentes (cinco com síndrome de Down e cinco típicos), o que torna o estudo não probabilístico, ou seja, os resultados aqui apresentados são válidos apenas para a amostra em questão e não podem ser generalizados, fato esse devido à dificuldade em coletar um tamanho de amostra maior.

Essa pequena amostra é decorrente da dificuldade de encontrar esses lactentes no município, bem como a dificuldade de avaliá-los nesta faixa de etária de três a quatro meses. De acordo com os dados levantados na Secretaria Municipal da cidade, dos lactentes nascidos vivos no período em que foi desenvolvido o estudo, menos de $6 \%$ dos prontuários levantados referiam-se a lactentes com síndrome de Down. Além disso, 33\% dos lactentes com síndrome de Down foram para cirurgia no perído da coleta.

\section{IMPLICAÇÕES CLÍNICAS}

De acordo com o estudo ficou demonstrado que a preferência podal pode ser observada aos três e quatro meses de idade em lactentes com síndrome de Down e típicos. Contudo, nota-se que pela diferença nas preferências podais dos grupos podemos supor que o processo de lateralização dos indivíduos com síndrome de Down é diferente dos lactentes típicos.

Além disso, foi constatado que na idade de três meses ambos os grupos sofrem influência do RTCA na preferência podal, mas que aos quatro meses somente o grupo com síndrome de Down evidenciando um atraso no desevolvimento nesse grupo, pois neste período esse reflexo não poderia se apresentar de forma tão evidente, influenciando a preferência.

Assim, o estudo é um parâmetro de triagem para identificar atraso no desenvolvimento de lactentes, o que, por sua vez, proporciona uma intervenção fisioterapêutica precoce. De acordo com os resultados, o presente estudo traz informações de grande benefício para a atuação fisioterapêutica tanto na avaliação como no acompanhamento e intervenção de lactentes com síndrome de Down e outros que apresentam disfunções motoras.

\section{CONCLUSÃO}

Conclui-se que lactentes com síndrome de Down apresentam uma preferência podal contrária aos lactentes típicos, sendo esta à esquerda ou indefinida, devido às alterações neuromotoras e de organização cerebral dos lactentes com síndrome de Down. Além disso, o peso adicional não influencia na preferência podal de ambos os grupos no período de três e quatro meses de idade, pois, para o grupo de lactentes com síndrome de Down existe a dificuldade na percepção do peso adicional pelos proprioceptores e, para os lactentes típicos, o peso foi insuficiente para estimular os proprioceptores. Em contrapartida, a preferência podal de ambos os grupos é influenciada pelo RTCA aos três meses de idade e somente o grupo de lactentes com síndrome de Down é influenciado aos quatro meses de idade. Com isso, constata-se que os lactentes com síndrome de Down apresentam atraso no desenvolvimento motor e a permanência do efeito do RTCA na preferência podal. No entanto, estas conslusões são restritas à amostra em análise.

\section{REFERÊNCIAS}

1. Sadeghi H, Allard P, Prince F, Labelle H. Symmetry and limb dominance in able-bodied gait: a review. Gait Posture. 2000;12(1):34-45.

2. Peters M. Footedness: Asymmetries in foot preference and skill and neuropsychological assessment of foot movement. Psychol Bull. 1988:103(2):179-92.

3. Piek JP, Gasson N. Spontaneous kicking in fullterm and preterm infants: Are there leg asymmetries? Hum Mov Sci. 1999;18(2-3):377-95.

4. Coren S, Porac C, Duncan P. Lateral preferences behaviors in preschool children and young adults. Child Develop. 1981;52(2):443-50. 
5. Peters M, Durding BM. Footedness of left-and right- handers. Am J Psychol. 1979;92(1):133-42.

6. Vaal J, van Soest AJ, Hopkins B. Spontaneous Kicking Behavior in infants: age-related effects of unilateral weighting. Dev Psychobiol. 2000:36(2):111-22.

7. Chen YP, Fetters L, Holt KG, Saltzman E. Making the mobile move: constraining task and environment. Infant Behav Develop. 2002:25(2):195-220

8. Thelen, E. Developmental origins of motor coordination: leg movements in human infants. Dev Psychobiol. 1985;18(1):1-22

9. Ângulo-Barroso RM, McKay SM. Longitudinal assessment of leg motor activity and sleep patterns in infants with and without down syndrome. Infant Behav Dev. 2006;29(2):153-68.

10. Schwartzman JS. Síndrome de Down. São Paulo: Memnon; 2003.

11. Silva N, Dessen M. Deficiência Mental e Família: Implicações para o Desenvolvimento da Criança. Psic Teor e Pesq. 2002;17(2):133-41.

12. Elliott D, Weeks, DJ, Chua R. Anomalous cerebral lateralization and Down Syndrome. Brain Cogn. 1994;26(2):191-5.

13. Carlier M, Stefanini S, Christine D, Volterra V, Doyen AL, Lamard C, et al. Laterality in Persons with Intellectual Disability. I- Do patients withtrisomy 21 and Williams-Beuren syndrome differ from typically developing persons? Behav Genet. 2006;36(3):365-76.

14. Gérard-Desplanches A, Deruelle C, Stefanini S, Ayoun C, Volterra V, Vicari S, et al. Laterality in Persons With Intellectual Disability II. Hand, Foot, Ear, and Eye Laterality in Persons with Trisomy 21 and Williams-Beuren Syndrome. Dev Psychobiol. 2006;48(6):482-91

15. Heriza CB. Implications of a dynamical systems approach to understanding infant kicking Behavior. Phys Ther. 1991;71(3):222-35.

16. Thelen E, Skala KD, Kelso JA. The dynamic nature of early coordination: Evidence from bilateral leg movements in young infants. Dev Psychobiol. 1987;23(2):179-86.
17. Ulrich BD, Ulrich DA, Ângulo-Kinzler R, Chapman DD. Sensitivity of Infants With and Without Down Syndrome to Intrinsic Dynamics. Res Q Exerc Sport. 1997;68(1):10-9.

18. Piek JP. The contribution of spontaneous movements in the acquisition of motor coordination in infants. In: Glencross DJ, Piek JP (Eds). Motor control and sensory motor integration: Issues and directions. North-Holland: Elsevier; 1995. p.199-230.

19. Bly L. Motor skills acquisition in the first year: An illustrated guide to normal development. Tucson: Therapy Skill Builders; 1994

20. Landgraf JF, Tudella E. Efeito do peso externo nos chutes espontâneos de lactentes nos primeiros dois meses de vida. Rev Bras Fisioter. 2008;12(6):468-75.

21. Carvalho RP, Tudella E, Barros ML. Utilização do Sistema Dvideow na Análise Cinemática do Alcance Manual de Lactentes. Rev Bras Fisioter. 2005;9(1):1-7.

22. Jeng S, Chen L, Yau KT. Kinematic analysis of kicking movements in preterm infants with very low birth weight and full-term infants. Phys Ther. 2002;82(2):148-59

23. Schneider K, Zernicke RF. Mass, centre of mass, and moment of inertia estimates for infant limb segments. J Biomech. 2002;25(2):145-8.

24. Pretchl HFR, Beintema DJ. The neurological examination of the fullterm newborn infant. Clin Dev Med. 1964:12:1-73.

25. Heath M, Grierson L, Binsted G, Elliott D. Interhemispheric transmission time in persons with Down syndrome. J Intellect Disabil Res. 2007;51(Pt 12):972-81.

26. Bryden MP. Choosing sides: the left and right of the normal brain. Canadian Psychol. 1990;31(4):297-309

27. Annett M. Handedness in families. Ann Hum Genet. 1973;37(1):93-105.

28. Lent, R. Cem bilhões de neurônios? Conceitos fundamentais de neurociência. São Paulo: Atheneu; 2010.

29. Meur A, Staes L. Psicomotricidade: educação e reeducação: níveis maternal e jardim. São Paulo: Manole; 1989. 